\title{
OKKASIONELLE LEXIK IN MEDIALEN TEXTEN. PRAGMALINGUISTISCH BETRACHTET AM BEISPIEL DER TEXTSORTE PRESSEKOMMENTAR
}

Der Beitrag behandelt am Beispiel der deutschsprachigen Pressekommunikation theoretischmethodologische Voraussetzungen pragmatisch orientierter Text- und Stilbeschreibung. So wird zunächst die Problematik des lexikalischen Okkasionalismus und seiner Definierbarkeit angesprochen, in einem nächsten Schritt ein pragmatisch orientiertes Modell zur Beschreibung und Analyse von Texten (Heusinger 1994) dargestellt und anschließend wird dieses modifiziert an drei ausgewählten Kommentartexten aus der deutschen überregionalen Presse angewandt, um zu zeigen, dass zum Erschließen des komplexen und vielfältigen funktional-stilistisch-pragmatischen Potentials okkasioneller Lexik eine aspektreiche, integrative, sogar interdisziplinäre Herangehensweise notwendig ist. Das angewandte Modell erweist sich dabei als geeignete methodologische Grundlage.

\section{Zum Begriff der okkasionellen Lexik}

Das Lexikon einer jeden Sprache lässt sich u.a. auf Grund der Dichotomie usuell/okkasionell differenzieren. Während auf der einen Seite für usuelle Lexik weitgehend Konsens darüber besteht, dass es sich um in einer Sprachgemeinschaft stabilisierte und daher überindividuell verfügbare und reproduzierbare lexikalische Einheiten handelt, sind auf der anderen Seite zum Teil unterschiedliche Auffassungen des Okkasionellen in der Lexik zu beobachten. Dies zeigt sich nicht zuletzt in der Vielfalt der lexikologischen Terminologie zur Benennung gelegentlicher lexikalischer Innovation, so z.B. Okkasionalismus, Neologismus, Gelegenheitswort, Textwort, Ad-hoc-Bildung, Einmalbildung, Neubildung, lexikalische Innovation u.a.m. Unterschiedliche Ausdrücke meinen dabei meist nicht differente Klassen der Lexik, sondern sie heben in der Regel nur verschiedene Schwerpunkte in der Beobachtungsperspektive hervor, nämlich jene, die die jeweilige Benennung auch motiviert haben.

Allen differenten Benennungen und zum Teil unterschiedlichen Begriffsauffassungen sind allerdings zwei wesentliche Merkmale gemeinsam: (1) das Merkmal der Neuheit und (2) das Merkmal der Nicht-Lexikalisiertheit. Neu ist dabei als neu im Vergleich zum bestehenden Lexikon zu interpretieren, zugleich aber als eine äußerst dynamische Größe und dementsprechend als eine graduell ausgeprägte lexikalische Eigenschaft zu verstehen. So ist sie bei den neuen Wörtern, die streng genommen einmal, spontan, ad-hoc und text-/kontextgebunden vorkommen und chronologisch als bisher nocht nicht vorhanden bzw. semantisch als isoliert nicht bekannt, nicht/weniger verständlich wahrgenommen werden, am stärksten ausgedrückt. Aus pragmalinguistischer bzw. handlungstheoretischer Perspektive sind Okkasionalismen in dieser Lesart Resultate individueller sprachlicher Handlungen, die in situativen Kommunikations- 
sowie Textzusammenhängen vollzogen werden. Daher kann die Bedeutung solcher Wörter im Prinzip nur über dieselben Zusammenhänge erschlossen werden, da sie auf der Systemebene der Langue fehlt. Oft genug ist derartige Lexik in literarischen Texten zu finden, wie z.B. einige Belege aus der Lyrik der Österreicherin Christine Lavant: Mitleidsaft, Nebelfrau, Mittagsfrau, Mondgras, herzverwelkt, warmgeträumt. Zusätzlich wirken solche Wörter auffällig, überraschend, da sie übliche kombinatorische Normen verletzen.

Die semantische Text-/Kontextgebundenheit ist jedoch nicht einfach eine generelle und obligatorische Eigenschaft der okkasionellen Lexik. Betrachtet man die neuen Wortbildungen - und wie die Auszählungen für das Deutsche gezeigt haben, überwiegen gerade Wortbildungsprodukte unter Okkasionalismen (vgl. u.a. Bußmann, 1990) - bezüglich ihrer Motiviertheit, so ergibt sich, dass viele davon problemlos auch losgelöst vom Text/Kontext semantisiert werden können. Begründet ist dies vor allem durch die Usualität einzelner Konstituenten, die solche Neubildungen morphologischsemantisch transparent machen. Dies gilt verstärkt für den Sprachgebrauch in den Medien, so auch für die meisten Wortbelege aus den beobachteten Texten, die sich im Anhang befinden. Hohe Produktivität einiger Wortbildungsmodelle im heutigen Deutsch (z.B. substantivische Determinativkomposita aus zwei substantivischen Konstituenten, die fast ohne Einschränkung entstehen) sowie starke Wortbildungsaktivität mancher usueller Lexeme, die sowohl als Erst- als auch als Zweitglieder in den Zusammensetzungen reihenbildend auftreten (wie z.B. Belege mit Nato, UN und UNO als Konstituente) tragen dazu wesentlich bei. ${ }^{1}$ Die Bedeutung vieler neuer Wortbildungen ist folglich auch ohne Bezugnahme auf den Text/Kontext, d.h. isoliert erschließbar, sogar erwartbar und voraussagbar. Somit ist anzunehmen, dass die Rezeption und wohl auch die Produktion hochgradig motivierter Wortneubildungen weitgehend automatisiert verlaufen und dass diese deshalb eher ausnahmsweise überhaupt noch als lexikalische Innovationen wahrgenommen, identifiziert und reflektiert werden (vgl. Matussek 1994, Barz 1996). Über eine besondere sprachliche Kreativität kann bei manchen davon nicht die Rede sein, so dass sie auch nicht über die Merkmale auffällig, überraschend, kombinatorische Normen verletzend verfügen.

Wie gesehen, operiert die Lexikologie, um okkasionelle von usueller Lexik zu unterscheiden, mit schwer definierbaren und sehr vagen, eher subjektiven Begriffen wie neu, auffällig, überraschend, bisher nicht bekannt, nicht/weniger verständlich, also mit Eigenschaften, die zudem hinsichtlich der Intensität graduell ausgeprägt und

1 Obgleich in den Komposita mit einer identischen Konstituente diese mit verschiedenen Bedeutungen beteiligt sein kann, wird die entsprechende Semantisierung - zumindest bei einem Muttersprachler - trotzdem in der Regel gewährleistet, und zwar durch seine Weit bzw. Sprachkenntnisse und -erfahrungen. So ist wohl real einzuschätzen, dass das Lexem Haus als Erstglied in den substantivischen Komposita entweder in der Lesart 'Gebäude' (Hausbesitzer, Hausnummer, Haustür, Hausverwalter) oder 'Familie' (Hausfreund, Hausarzt) oder 'zu Hause' (Hausaufgabe, Hausmantel, Hausschuh) oder 'Firma, Institution' (Hausjurist, Hausmitteilung) oder 'Haushalt' (Hausfrau, Hausmann, Hausmüll) verstanden wird. 
stark auf die Sprachkompetenz des Beurteilers angewiesen sind. Aus der Sicht eines Nichtmuttersprachlers ist die Situation naturgemäß wiederum anders, sogar viel komplizierter. Aus diesem Grund wird im Folgenden bewusst vereinfacht und als okkasionell diejenige Lexik aufgefasst, der im Allgemeinen das Merkmal der Neuheit und das Merkmal der Nicht-Lexikalisiertheit zuzuschreiben sind. Neu wird dabei nicht als zum ersten Mal auftretend verstanden, da dies praktisch überhaupt nicht feststellbar ist, und nicht lexikalisiert ist als nicht lexikographisch kodifiziert zu lesen. Die Unterscheidungskriterien zwischen usuell und okkasionell zielen somit hauptsächlich auf die Qualitäten neu im Vergleich zum bestehenden Lexikon sowie sozial nicht stabilisiert/ verbreitet und weniger auf die Eigenschaften auffällig und/oder semantisch text-/kontext-gebunden $\mathrm{ab}$. Um sich nicht nur auf individuell-subjektive intuitive Entscheidungen stützen zu müssen, wurde die als okkasionell eingestufte Lexik bezüglich der lexikographischen Kodifizierung geprüft, und zwar am achtbändigen großen Duden-Wörterbuch, da dieses, laut Begründung der Wortauswahl in Band 1, eben "individualsprachliche Prägungen und Augenblicks- oder Situationsbildungen, d.h. Wörter, die jederzeit gebildet werden können, die aber nicht fester Bestandteil unseres Wortschatzes sind" (Duden 1993, 7) unberücksichtigt lässt. Auch die neueste elektronische Ausgabe des Duden Universalwörterbuchs (2001) verzeichnet die beobachtete Lexik nicht.

Wichtig ist allerdings, dass die fehlende lexikographische Kodifizierung eines Wortes noch kein Beweis für seine fehlende Usualisiertheit und Lexikalisiertheit, also auch für seine Okkasionalität sein muss. Die Kategorie neu wird somit nicht streng frequenzbezogen und chronologisch im Sinne von einmalig bzw. erst seit kurzem vorhanden aufgefasst, sondern eventuell auch als rezeptiv verständlich, jedoch nicht überindividuell, üblich, verbreitet interpretiert. Die beobachteten Okkasionalismen sind demnach nicht unbedingt Einmalbildungen. Zudem wurden sie dem Massenmedium Zeitung entnommen, so dass es sich entweder um solche Neuerungen handelt, die angenommen für den konkreten Text gebildet worden sind (wie z.B. Balkanaktion, Balkanpolitik, Zufallskoalition in (1), Bosnien-Mission, Bosnien-Politik, UN-Mission, Befehlskette in (2), Winterfrieden, Kriegswinter, Friedenswinter in (3), wohl aber auch um solche, die aus dem individuellen Wortschatz eventuell schon herausgetreten sind, die eine zeitlich und thematisch begrenzte Aktualität in den Medien und somit einen bestimmten Grad der Usualisiertheit zwar nachweisen können, jedoch mit lexikalisierten Wörtern (noch) nicht gleichzusetzen sind (wie z.B. Nato-Staat, Nato-Truppe, NatoSoldat, Uno-Blauhelm, Balkankrieg, Nato-Mitglied in (1), Nato-Gipfel, Nato-Land, UN-Sicherheitsrat in (2), NATO-Friedenstruppe, Rest-Jugoslawien in (3). Möglicherweise können einige auch als auffällig wahrgenommen werden, z.B. Krisenmanager, Grünhelm, Zufallskoalition in (1), Friedensdienstleister, Befehlskette in (2), Winterfrieden, Friedenswinter, europäisches Beirut in (3). 


\section{Okkasionelle Wörter im Text}

Eine weitere und für folgende Ausführungen wesentliche Eigenschaft von Okkasionalismen ist, dass sie weitgehend sekundäre Benennungen sind, also meist Konkurrenzbenennungen zur bereits existierenden Lexik. Als eine Art Prestige- bzw. Luxuswörter gehen sie in erster Linie aus konkreten jeweiligen situativ-kommunikativen Bedürfnissen hervor und erfüllen seltener das elementare kommunikative Verlangen nach der Benennung. Umso mehr interessieren deshalb sprecherseitige Motive, die zu ihrer Bildung/Verwendung führen sowie ihre möglichen Leistungen und Auswirkungen, kurzum Funktionen im Text. Einen akzeptablen Zugang an stilistische, pragmatische und sonstige funktionale Potentiale okkasioneller Lexik sichern kommunikativ-pragmatisch ausgerichtete methodologische Untersuchungsansätze, die in der neueren Sprachwissenschaft auf Analyse und Interpretation sprachlicher Kommunikation an sich angewandt werden.

Versteht man die Textgestaltung und die Textrezeption als sozial-kommunikative und intentionale Tätigkeiten und den Text als intentionsgesteuerte und zielgerichtete Äußerung, als Instrument kommunikativen Handelns mit einer komplexen Handlungsstruktur, so ist seine sprachliche Ausprägung nicht nur auf die Proposition des Textes zurückzuführen. Die Verwendung der okkasionellen Lexik ist somit nicht einfach als Resultat der Bemühungen um einen interessanten, guten Stil zu interpretieren, sondern die jeweilige Entscheidung über die okkasionelle (sowie usuelle) Lexik unterliegt haupsächlich der intentionalen Textsortenkomponente und/oder der gesamten kommunikativen Situation. Von besonderem Interesse sind somit Zusammenhänge zwischen kommunikativen Handlungsstrukturen und ihrer sprachlichen Widerspiegelung im jeweiligen Text. Handlungstheoretisch orientierte Untersuchungsansätze scheinen diese akzeptabel und weitgehend verdeutlichen zu können. Handlungsorientiert ist auch das Textbeschreibungsmodell von Heusinger (1995), welches im Folgenden in seinen Wesenszügen dargestellt und anschließend - allerdings modifiziert - als Grundlage einer Analyse und Interpretation der okkasionellen Lexik in der Pressetextsorte Kommentar erläutert wird.

Die methodologische Basis dieses Text- und Stilbeschreibungsmodells ist interdisziplinär angelegt und bezieht sprachlich-kommunikative Aspekte funktionaler, textstruktureller und pragmatisch-stilistischer Art mit ein. Modellgerecht werden am jeweiligen Text schrittweise folgende Aspekte verfolgt: (1) Handlungscharakteristika, (2) allgemeine sprachliche Eigenschaften, (3) spezielle sprachliche Charakteristika und (4) Strukturcharakteristika.

Unter den Handlungscharakteristika (1) werden mehrere beeinflussende Größen der sprachlichen Kommunikation thematisiert: Kommunikationsabsicht, Handlungstyp, Kommunikationsgegenstand und Kommunikationssituation. Aus handlungstheoretischer Perspektive erweist sich die Textgestaltung, wie gesagt, als hochgradig bewusst vollzogene, intentionsgeleitete und zielgerichtete kommunikative Tätigkeit. Mit dem textuellen Handlungstyp wird "die Gesamthandlung bestimmende sprachlich- 
kommunikative Handlung angezeigt, mittels der der Text hervorgebracht worden ist" (Heusinger 1995, 120). Von grundlegender Bedeutung sind dabei konkrete Handlungsstrategien, die sich u.a. auch in der Selektion geeigneter lexikalischer Mittel zur Realisierung der kommunikativen Ziele zeigen. Somit betrifft die Auswahl der Lexik sowie ihre Anordnung und Verflechtung im Text nicht nur und nicht einfach die propositionale Text-Komponente, auch befolgt sie nicht nur Prinzipien der sprachlichen Wohlgeformtheit. Sie beruht weitgehend auf den sprechereigenen Absichten, d.h. auf sprechereigenen individuellen Meinungen und kommunikativem Willen, zugleich aber auch auf der Textintention der realisierten Textsorte, d.h. im Sinne davon, als was eine Text-Äußerung in der gegebenen Situation gilt/gelten kann. Die intentionale Komponente sprachlicher Kommunikation ist demnach eine psychologische, individuell geprägte Dimension und ebenso eine sozial bedingte Größe. Sie erstreckt sich von den individuellen Absichten des Sprechers bis zu den überindividuell gültigen und konventionalisierten Textsortenintentionen. Zudem wird von beiden Kommunikationspartnern erwartet, dass sie soziale Konventionen, eingespielte soziale Regeln in der sprachlichen Kommunikation sowie konventionalisierte Textsortenintentionen zumindest in ihren Wesenszügen kennen und beim Verfassen bzw. Erschließen von Texten auf sie zurückgreifen. Dadurch werden sie sich auch der gesamten jeweiligen kommunikativen Situation stärker bewusst.

In Bezug auf allgemeine sprachliche Eigenschaften (2) interessieren eventuelle Abweichungen von der jeweils erwarteten Sprachvarietät. Wo z.B. der Standard erwartet wird, lassen sich umgangssprachliche und/oder dialektale Besonderheiten weitgehend als stilistische Auflockerungsmittel und als Indikator von Stilschichten interpretieren.

Allgemeine sprachliche Eigenschaften sind aufs Engste mit den speziellen lexikalischen bzw. stilistischen Charakteristika (3) verbunden. Es geht dabei um genuin linguistische Fragestellungen an grammatische, lexikalische und stilistische Eigenschaften des Textes. Bezogen auf Okkasionalismen wird ihr funktionales Leistungspotential angesprochen, d.h. Motivation zu ihrer Bildung/Wahl auf Seiten des Sprechers sowie eventuelle Wirkungen auf Seiten des Rezipienten. Verschiedenartige grammatische und/oder lexikalische Besonderheiten gelten als Abweichungen vom Erwarteten und sind zugleich Stilelemente.

Unter Strukturcharakteristika (4) werden Textkomposition, Kanal und Art der Äußerung sowie Textstruktur im Sinne von Textkohärenz bzw. Textverflechtung berücksichtigt. Wenn man davon ausgeht, dass geschriebene Texte sehr oft Muster- Realisierungen und als solche einzelnen Textsorten zuzuordnen sind, ist es meist möglich, Prinzipien der inhaltlichen Textgliederung bzw. Elemente der textuellen Struktur zu rekonstruieren. Der Kanal und die Art der Äußerung sind insofern von Bedeutung, als bei geschriebenen Texten Nonverbales als eventueller Träger von zusätzlichen Informationen und/oder Verstehensanweisungen fehlt. Folglich sollen diese explizit sprachlich zum Ausdruck kommen. Das Phänomen der Textkohärenz macht es möglich, semantisch-kognitive Zusammenhänge im Text zu entdecken. 
Ein Textbeschreibungs- und Textinterpretationsrahmen wie das dargestellte Modell Heusingers ermöglicht eine komplexe und integrative Beschreibung des Textes und seines Stils, und zwar differenziert nach verschiedenen Parametern. Dabei können einige Untersuchungsaspekte dominieren und andere wiederum in den Hintergrund treten. Auf die okkasionelle Lexik zielen modellgerecht besonders spezielle sprachliche Charakteristika (3) ab, denn ein okkasioneller Ausdruck, als sekundäre Benennung meist Alternative zur bereits existierenden Lexik, ist im Prinzip ein Spezifikum.

\section{Okkasionelle Wörter im Kommentar}

Postulate des dargestellten Modells werden im Folgenden auf den Pressekommentar als klassischer Vertreter meinungsbetonter medialer Texte übertragen. Die analysierten Kommentartexte wurden anlässlich der Friedensverhandlungen in Dayton in der deutschen überregionalen Presse veröffentlicht, um geplante Interventionen, internationale Militäreinsätze während des Krieges auf dem Balkan wie auch die Nato als Initiator dieser Aktionen zu kommentieren. Das Modell wird allerdings modifiziert, und zwar werden jene Modellaspekte in Betracht bezogen, die sich bei der Beobachtung und Interpretation okkasioneller Lexik als ergiebig erweisen mögen. Das sind: (1) der situative Kontext der Kommunikation im weiteren Sinne, d.h. Handlungscharakteristika, (2) das pragmatisch-stilistische Potential der Lexik und (3) Struktureigenschaften der realisierten Textsorte.

\section{$\mathrm{Zu}$ (1): Der situative Kontext}

Situative Einflussfaktoren in der Pressekommunikation sind materieller Art, bezogen auf die Sender-Text-Empfänger-Konstellation sowie nicht-materieller Art, verweisend auf die gesamte Wahrnehmung des Situativen beim Rezipienten, also Kommunikationssituation, Kommunikationsgegenstand, Kommunikationsabsicht und Handlungstyp.

Ist der Rezipient ein regelmäßiger Zeitungsleser, so ist ihm weitgehend bekannt, dass ein Kommentartext als Textsortenmusterrealisierung in der Zeitung vorkommt und über spezifische Textsorteneigenschaften verfügt. Aus Erfahrung mit der Presse weiß er auch, dass Kommentar als Textsorte nicht isoliert sondern in eine umfangreichere Berichterstattung über öffentlich relevante Themen integriert vorkommt. Auch kennt er die Tätigkeit des Kommentierens als Verhaltensmuster aus seinem Alltag, Stellungnahmen, Meinungsäußerungen, Bewertungen kommen nämlich in alltäglicher Kommunikation oft genug vor. Dementsprechend rechnet der Kommentator beim Leser mit gewissen Vorkenntnissen zum kommentierten Thema wie auch mit entsprechendem Kontextwissen und berücksichtigt dies bei der Textgestaltung. Auf den ersten Blick befindet sich der Kommentator dabei in privilegierter Position. Er verfügt über mehr Informationen und hat bei der Textgestaltung im Prinzip mehr Freiraum als der Leser bei der Rezeption/Interpretation. Eine gewisse Einseitigkeit in konkreten Autor-Leser-Beziehungen im Fall Presse ist jedoch zu relativieren, denn 
eher sind Beide wechselseitig aufeinander bezogen. Zumindest sprechen dafür zwei Tatsachen: die Presse ist Ware und der Leser entscheidet sich selbst für die Lektüre oder er lehnt sie ab. Hierbei wirft sich die Frage auf, ob und wenn ja, inwiefern der Autor das sprachliche Bild seiner Texte an den angenommenen Leser anpasst. Befolgt er auch in sprachlicher Hinsicht das so genannte "Prinzip der Antizipation möglicher Rezipientenreaktionen" (zit. nach Jesenšek 1998, 142), wie in der einschlägigen Literatur zur medialen Kommunikation zu lesen ist? Mit welcher Absicht und Funktion erscheinen nun Okkasionalismen im Text? Funktionieren sie verständnisfördernd, wird also bei ihrer Bildung und Verwendung der potentielle Leser beachtet oder führt ihre Einsetzung im Text möglicherweise, bewusst oder unbewusst auch zu Missverständnissen in der intendierten Lesart?

Wie aus den Exemplartexten ersichtlich (Texte (1), (2) und (3) im Anhang), überwiegen unter Okkasionalismen substantivische Determinativkomposita, bestehend aus eher nicht überraschenden, erwartbaren Kombinationen usueller Lexik. In ihren internen semantischen Beziehungen sind sie weitgehend transparent und somit im Wesentlichen problemlos dekodierbar, noch insbesondere, wenn sie an einigen Stellen auch anaphorisch oder kataphorisch eingeführt bzw. erklärt werden (wie in (1): auf dem Balkan soll die Nato den Frieden sichern (im Untertitel), im Nachtext FriedensSicherungstruppe, oder in (3): ein geplanter Gebietsaustausch /.../: Die kroatische Halbinsel Prevlaka /.../ gegen Hinterland von Dubrovnik). Für die meisten beobachteten Wortbelege kann man sagen, dass sie unauffällig und weitgehend verständlich sind, so dass sie deshalb eher ausnahmsweise wenn überhaupt als neue okkasionelle Lexik empfunden werden. Eine Ausnahme in dieser Hinsicht mögen etwa die Ausdrücke Krisenmanager, Grünhelm in (1) oder Winterfrieden, Friedenswinter, europäisches Beirut in (3) sein, da sie eine bewusstere Dekodierung, mehr Engagement und mehr notwendige Hintergrundinformationen vom Leser verlangen. Allerdings lässt sich sagen, dass die zitierten Wortbelege für den Leser im Wesentlichen kein Hindernis für die intendierte Rezeption der Kommentare sind und dass sie eher verständnisfördernd und sogar interpretationssteuernd wirken. Wohl im Dienste der generellen, nicht unbedingt nur kommentarspezifischen kommunikativen Intention: Äußerungen so zu versprachlichen, dass sie verstanden, akzeptiert und möglicherweise auch übernommen werden.

Hinsichtlich des Kommunikationsgegenstands, der Textproposition als semantische Dimension des Textes lassen sich manche Okkasionalismen in einzelne Topik-Ketten einordnen (vgl. Topik-Ketten zu den Texten (1) und (2) im Anhang). Sie partizipieren ausgiebig an der Topik-Realisierung, und zwar als variante bzw. konkurrierende Benennungen zu den voraus- oder nachgehenden Lexemen und Syntagmen. Mit diesen sind sie referenzidentisch, sie koreferieren auf denselben Sachverhalt und zeigen somit Inhalts- und Sinnzusammenhänge im Text an, als Mittel der Topikalisierung tragen sie zur Textkohärenz bei. Ihre Beziehungen zueinander und zu den usuellen Isotopiegliedern sind jedoch nur anscheinend synonymer Art. Als Benennungen sekundärer Art 
bringen sie notwendigerweise weitere/andersartige Aspekte in der Benennung hervor. Demnach sind sie nicht als bloße Substituentia ohne zusätzliche semantische bzw. stilistisch-pragmatische Auswirkungen aufzufassen.

Weiterhin ist in Bezug auf die Textproposition eine textverflechtende Funktion von Okkasionalismen zu beobachten. Die Textverflechtung wird morphologisch oft durch Wiederholung identischer Grundmorpheme in aufeinander folgenden Wortbildungen gewährleistet. Das wiederholte Grundmorphem, als Wortbildungskonstituente oder auch frei auftretend, wie etwa Nato oder Frieden in Text (2) (vgl. Okkasionalismen in textverflechtender Funktion im Anhang), leistet, trotz der eventuellen Referenzunterschiede, die inhaltliche Zusammengehörigkeit der betreffenden Wörter, da notwendigerweise an der jeweiligen Gesamtbedeutung beteiligt. Die Entfaltung des Textthemas wird dadurch eindeutiger, die Textorganisation überschaubarer. Auch von der Rezipientenseite her scheint es, dass dadurch textuelle Sinnzusammenhänge leichter zu erfassen sind. So kommen textuelle Funktionen von okkasionellen Bildungen verstärkt zur Geltung. Folglich sind Selektionskriterien, auf Grund derer sich der Autor für einzelne Konstituenten/Komponenten seiner Wortneubildungen entscheidet, auch textueller bzw. textkompositioneller Art.

Die intentionale Textkomponente lässt sich folgendermaßen interpretieren: (1) als individuelle/subjektive Sprecher-Absichten und (2) als konventionalisierte Textsortenintentionen. Die Ersteren führen den Sprecher/Autor u.a. dazu, dass er unter möglichen Vertextungsformen (Textsorten) diejenige auswählt, deren konventionalisierte Textsortenintentionen den individuellen Absichten entsprechen. Die Textsortenintention eines Kommentars ist hauptsächlich darin zu suchen, dass die autoreigenen Stellungnahmen samt obligatorischen Bewertungen vom Leser identifiziert, verstanden, akzeptiert und möglicherweise auch übernommen werden. Der Kommentator bemüht sich, an den Leser zu appelieren, und das tut er, indem er eine entsprechende Textsorte wählt (Kommentar, dem Appelieren insgesamt als Textsortenintention zuzuschreben ist) und indem er im Prozess der Textgestaltung einzelne sprachliche Mittel intentionsgeleitet, d.h. strategisch einsetzt. Möglicherweise sind diese Mittel auch Okkasionalismen, an denen sich das Intentionale im Sinn beider Auffassungen manifestieren kann. Immerhin ist an Beispieltexten (1) und (2) z.B. nicht zu behaupten, dass das Textsortenintentionale isoliert an zitierten Okkasionalismen interpretierbar wäre, zumal sie mit wenigen Ausnahmen keine Bewertungen in die Kommunikation mitbringen. Anders allerdings in Text (3). Die Wortbildung Winterfrieden (im Titel) lässt durch das Assoziationspotential der Konstituenten bewertende Einstellungen zum kommentierten Thema annehmen. Gleich nach der Einführung der Text-Proposition (Einsatz der Nato im ehemaligen Jugoslawien, im ersten Satz) bezweifelt der Kommentator das Vorhaben der Nato in Bosnien und weist auf Fährnisse hin. Diese werden im darauffolgenden Text spezifiziert, zusätzlich erläutert und der Zweifel an geplanter Intervention kulminiert in der textabschliesenden Behauptung: Der erste Friedenswinter aber wird in vielem noch vier Kriegswintern gleichen. Das assoziative Potential des Lexems Winter in 
Winterfrieden und Friedenswinter ('kalt', 'dunkel', 'hart', auch 'Abkühlung in den Beziehungen') unterstützt die Bezweiflung und Problematisierung des Friedens. Die beiden okkasionellen Wörter indizieren die Absicht des Kommentators und bekräftigen zugleich die appelative Textsortenintention. Okkasionalismen sind also möglicher weise Indizien für kommunikative Absichten des Sprechers und korrelieren oft mit konventionalisierten Textsortenintentionen.

Mit der Intention eng verbunden ist der Begriff des Handlungstyps. Handlungstheoretischem Textverständnis entsprechend ist der jeweilige Kommentartext Ergebnis meist mehrerer sprachlich-kommunikativer Handlungen. So ist seine textuelle Struktur ein Komplex hierarchisch verknüpfter Sprachhandlungen, unter denen das Bewerten dominiert. Untergeordnete Handlungen mit dem dominanten informierenden und/oder argumentierenden Charakter wie z.B. Mitteilen, Feststellen, Behaupten, Begründen, Widerlegen, Vergleichen u.ä. sollen zur Akzeptabilität der zentralen Bewertung beitragen und sie unterstützen. Das Bewerten ist somit der Handlungstyp, der dem Kommentar zugrunde liegt und der die gesamte Handlungsstruktur von Kommentartexten beherrscht. Sind nun Okkasionalismen möglicherweise Indizien sprachlich-kommunikativer Handlungen? Oder: Entscheidet sich der Kommentator für ihre Bildung und Verwendung auch, um mit ihrer Hilfe kommentarspezifische Handlungen wirkungsvoller zu vollziehen? Wie gesehen, zeigt das Wort Winterfrieden in (3) durch das assoziative Potential der ersten Konstituente die kommunikativen Absichten des Kommentators an. Und da der Textintention zielgerichtete sprachliche Handlungen zugrunde liegen, ist Winterfrieden in (3) wohl auch als Anzeichen für eine im Text vollzogene Bewertungshandlung interpretierbar. Man kann also davon ausgehen, das okkasionelle Bildungen eventuell assoziativ-konnotative bewertende Komponenten enthalten, z.B. Friedenswinter, Winterfrieden, das europäische Beirut, Rest-Jugoslawien in (3), wodurch sie als Indikatoren der kommentarspezifischen Handlungstypik erklärbar sind.

$\mathrm{Zu} \mathrm{(2):} \mathrm{Das} \mathrm{pragmatisch-stilistische} \mathrm{Potential} \mathrm{der} \mathrm{Lexik}$

Grundlegende Merkmale eines Okkasionalismus sind das Merkmal der Neuheit und das Merkmal der Nicht-Lexikalisertheit (vgl. Kap. 1). Die Kategorie neu im Vergleich zum bestehenden Lexikon müsste im Prinzip zugleich auffällig bedeuten, jedoch ist dies nicht unbedingt der Fall, wie manche Wortbelege in (1), (2) und (3) zeigen. In pragmatisch-stilistischer Hinsicht interessieren jedoch in erster Linie so genannte auffällige Wörter, also diejenige Lexik, bei der der Neuheitseffekt stärker ausgeprägt ist. Das sind etwa Wörter mit entlehnten und/oder fachsprachlichen Konstituenten/Komponenten bzw. weniger erwartete Konstituenten-Kombinationen, z.B. Krisenmanager, Grünhelm, Weltgendarm in (1), Nato-Friedensexpedition in (2), Friedenswinter, Winterfrieden in (3) - hier trägt das Spiel mit den Lexemen Frieden und Winter zur Auffälligkeit bei. Als relativ auffällig gelten auch Wörter, deren Konstituenten über das assoziative und emotionale Potential verfügen (etwa Grünhelm 
in (1), Bosnien-Mission, UN-Mission, Nato-Friedensexpedition in (2). Die Entscheidung über den Einsatz solcher Wörter ist demnach auch eine stilistische. Sie fungieren als lexikalische Stilelemente, sie sind Merkmale des Individualstils. An der beobachteten Lexik lassen sich zwei pragmatisch-stilistische Aspekte aussondern: (1) der Aspekt der expressiven Hervorhebung und (2) der Aspekt der syntaktischen Komprimierung.

Bezogen auf den Ersteren kommen nur so genannte auffällige Okkasionalismen zur Geltung, wie angenommen die Bildungen mit Mission und Expedition in (2): BosnienMission, UN-Mission, Nato-Friedensexpedition. Die Konstituente Mission wirkt wegen der Gebundenheit an Religion, Diplomatie eher bildungssprachlich, gehobener, während Expedition die semantische Komponente 'Abenteuerlichkeit' hervorruft. Durch implizierte Bewertungen wirken derartige Wörker verstärkt expressiv.

Der Aspekt der syntaktischen Komprimierung meint, dass Wörter als ökonomischere Einheiten entsprechende längere syntaktische Strukturen ersetzen können. Dies ist für manche der beobachteten Wortbelege festzustellen, z.B. FriedenssicherungsTruppe vs. Truppe mit der Aufgabe, Frieden zu sichern, Blauhelmaufgabe vs. Aufgabe der Blauhelme, Balkanaktion vs. Aktion auf dem Balkan, Zufallskoalition vs. Koalition, die zufällig zustande gekommen ist, um einige aus Text (1) zu nennen. Zumal eben in der Presse die Sprachökonomie eine wichtige Rolle bei der Textgestaltung spielt, fungieren sehr viele Okkasionalismen als bevorzugter Ersatz längerer Wortgruppen und anderer aufwendiger syntaktischer Strukturen. Im Deutschen ist dies durch die hohe Produktivität und Vielfältigkeit des Wortbildungssystems ermöglicht, insbesondere im Bereich der substantivischen Komposition. In rezeptiver Hinsicht scheinen solche Wörter eher unproblematisch zu sein, noch besonders, wenn sie aus geläufigen, usuellen Komponenten bestehen und/oder die Bildung und entsprechende Semantisierung sich durch anaphorische und/oder kataphorische Bezüge herleiten lässt, wie oben bereits erwähnt.

\section{Zu (3): Strukturcharakteristika der realisierten Textsorte}

Aus textlinguistischer Sicht sind Texte weitgehend Realisierungen konventionalisierter Textmuster mit typischer Textkomposition. In diesem Sinn befolgt auch jeder Kommentartext, zumindest in seinen Wesenszügen, ein konventionalisiertes prototypisches Muster mit spezifischen kommunikativen Aufgaben. Wie angedeutet, setzt das Wesen des Kommentierens die Sprachhandlung Bewerten voraus, die auch die kommentarspezifische textuelle Struktur dominiert. Nach den textlinguistischen Erkenntnissen ist das Bewerten in doppelter Hinsicht interpretierbar: (1) Bewertungen sind Bestandteile von kommentierenden Sprachhandlungen und/oder (2) Bewertungen resultieren aus den Bemühungen des Autors um einen wirkungsvollen, situationsgemäßen Stil seines Textes. Einerseits befolgen sie strukturinterne Prinzipien der Textmusterrealisierung, andererseits korrelieren sie mit strukturexternen, stilistischpragmatischen Prinzipien der Textgestaltung an sich. Auch unter Okkasionalismen lassen sich solche identifizieren, die Bewertungsträger an sich sind und die dadurch 
das Bewerten als obligatorische und dominante Textstrukturkomponete des Kommentars realisieren und/oder unterstützen.

So kann bei meheren beobachteten Wortbelegen eine bewertende Semantik vermutet werden, z. B. bei Weltgendarm, Zufallskoalition in (1), ebenso bei Bildungen mit Mission und Frieden als Konstituente in (2) und (3). Allerdings gründet sie zunächst auf assoziativem Gehalt einzelner Konstituenten und erst dann auf bewusster Wahrnehmung des Bewertens als obligatorische Komponente der Kommentartextstruktur. Die Lexeme Mission, Expedition, Zufall lösen bewertende Assoziationen aus, Mission etwa 'positiv', 'wichtig', 'ernst', Expedition 'unbekannt', 'gefährlich', Zufall 'unernst'. Auch lässt sich sagen, dass meist nur diejenigen Okkasionalismen auch explizite Bewertungsträger sind, die zugleich als auffällig eingestuft werden können.

\section{Schlusswort}

Mehrere differente Beobachtungsaspekte einer pragmatisch orientierten Text- und Stilbeschreibung, wie am dargestellten und modifizierten Modell Heusingers (1994) illustriert, führen zum Schluss, dass okkasionelle Lexik in funktional-pragmatischer Hinsicht ein komplexes Phänomen ist. Ihr semantisch-syntaktisches Potential erstreckt sich von der Funktion der (varianten) Benennung über kohärenzsichernde und verflechtende textuelle Funktionen bis zu den pragmatisch-stilistischen Leistungen, alles aufs Engste auf die jeweilige kommunikative Situation ihrer Verwendung bezogen. Einzelne Funktionen lassen sich voneinander nicht strikt und deutlich abgrenzen, ebenso wenig sind sie vom Text/Kontext isoliert zu beobachten. Deshalb ist für ihre Deutung und/oder Interpretation eine integrative Beobachtung notwendig. Das dargestellte Beschreibungsmodell scheint dieser Aufgabe gerecht zu werden.

\section{Literatur}

BARz, Irmhild (1996): Die Neuheit von Wörtern im Urteil der Sprecher. In: Hertel, V. et al. (Hg.): Sprache und Kommunikation im Kulturkontext. Beiträge zum Ehrenkolloquium aus Anlaß des 60. Geburtstages von Gotthard Lerchner. Frankfurt am Main, 299-314.

BußMANN, Hadumod (1990): Lexikon der Sprachwissenschaft. 2. Aufl. Stuttgart.

Duden Das große Wörterbuch der deutschen Sprache in acht Bänden (1993-1995). Mannheim etc.

Duden Deutsches Universalwörterbuch (2001). CD-ROM-Ausg. Mannheim etc.

FLEISCHER, Wolfgang, Irmhild BARZ (1995): Wortbildung der deutschen Gegenwartssprache. Unter Mitarbeit von M. Schröder. 2., durchg. u. erg. Aufl. Tübingen.

HeuSNGER, Siegfried (1995): Pragmalinguistik. Texterzeugung, Textanalyse; Stilgestaltung und Stilwirkungen in der sprachlichen Kommunikation. Ein Lehr- und Übungsbuch. Frankfurt am Main.

JESENŠEK, Vida (1998): Okkasionalismen. Ein Beitrag zur Lexikologie des Deutschen. Maribor.

Langenscheidts Großwörterbuch Deutsch als Fremdsprache (2001). Berlin etc.

LüGER, Heinz-Helmut (1995): Pressesprache. 2., neu bearb. Aufl. Tübingen.

MatusSEK, Magdalena (1994): Wortneubildung im Text. Hamburg. 


\section{Anhang}

(1) Krisenmanager, Balkanaktion, Weltgendarm, westliche Allianz, „Friedenssicherungs-Trupper, Nato-Staat, Balkanpolitik, Nato-Truppe, Uno-Blauhelm, Grünhelm, Nato-Soldat, Blauhelmaufgabe, Bündnis-grenze, zusammenschmieden, Nato-Mitglied, Balkankrieg, Zufallskoalition, Nato-Operation, Kooperationsgremium, Kansultationsgremium, westliches Bündnis;

(2) Nato-Soldat, Kriegspartei, atlantische Allianz, Bosnien-Mission, Nato-Land, Bündnisgrenze, Nato-Gipfel, UN-Mission, Friedensdienstleister, Befehlskette, UN-Sicherheitsrat, Nato-Friedensexpedition, Rosnien-Politik;

(3) Winterfrieden, Kriegswinter, NATO-Friedenstruppe, UNO-Blauhelm, Serbenführer. Serbenführung, "europäisches Beiruti, Gebietaustausch, Marinehafen. Rest-Jugoslawien, Wochenanfang, Friedenswinter,

Okkasionelle Lexik aus Texten (1) bis (3) 
(1) auf dem Balkan soll die Nato den Frieden sichern - die Balkanakion ${ }^{182}$ - die Aktion - massives gemeinsames Eingreifen - das Vorhaben - militärische Aufgaben jenseits der Bündnisgrenzen - die bevorstehende Balkanaktion - die Nato-Operation - militärische Kooperation auf dem Balkan - die Akrion in Bosnien - das Vorhaben der Nato - die Aktion - die Herausforderung im ehemaligen Jugoslawien

(2) Die Nato in Bosnien - das größte milizärische Unternehmen ihrer 46jährigen Geschichte - die Bosnien-Mission - UN-Mission - die erste in einer langen Reihe künftiger Nato-Friedensexpeditionen - derlei Unternehmen - "Krieg für den Frieden« - der Interventionismus - das Eingreifen - die Intervention - das militärische Mirtel

(1) schwerbewaffnete Einheiten - auch Truppen anderer Staaten - die jeczt geplante schwerbewaffnete "Friedenssicherungs-Trupper von 60000 Mann - eine Nato-Truppe - Grünhelme - die Nato-Soldaten

(2) Die Nato in Bosnien - die Vorhut von einigen zigtausend Nato-Soldaten - das Expeditionskorps der westlichen Allianz - die Friedenstruppe

(1) die Nato - das mächrigste Bündnis der Welt - die atlantische Allianz die Nato - eine neue Nato - das Bündnis als Krisenmanager in und um Europa - die Nato - schlüpft in die Rolle des Weltpolizisten - ein Bündnis - die fröhliche Übernahme der Aufgaben eines Weltgendarmes - die westliche Allianz - die sechzehn Nato-Staaten - die westliche Allianz Naro-Mitglieder - der Westen - die Nato - westliches Bündnis - die Nato - das Bündnis - die Nato - der Westen - das Bündnis - eine funkrionicrende Nato - das Bündnis

(2) Bündnis ohne Grenzen - die Nato - die westliche Allianz - die atlantische Allianz - das Bündnis - die Allianz - die Nato - Friedensdienstleister im früheren Jugoslawien - das Bündnis - die Nato - die Nato - das westliche Bündnis

Okkasionalismen als Mittel der Topikalisierung, Text (1) und (2) 
(2) Die Nato in Bosnien /.../; In den nächsten Tagen wird die Vorhut von einigen zigtausend Nato-Soldaten nach Bosnien aufbrechen; Artikel $S$ des Nordarlantikpakres begrenzre das Verteidigungsareal auf das Territorium der Nato-Länder, Beiträge zu weltweiter Stabilitär und welrweitem Frieden wurden auf dem Nato-Gipfel Ende 1991 beschlossen 1.../; Die Nato wurde damit entgrenzt; Ein Mandat des UN-Sicherheitsrates wird nur den papierenen Paravent abgeben, hinter dem die Nato in eigener Zuständigkeit /.../ handelt; Ist Bosnien die erste in einer langen Reihe künfriger Nato-Friedensexpeditionen rund um den Globus?; Wie viele "Kriege für den Frieden« soll die Naro führen?

(2) Das Expedicionskorps der westlichen Allianz, /.../ soll den heiklen Frieden verbürgen I.../; Im Rätseln darüber, I.../ was 1996 alles auf die Friedenstruppe zukommen mag /..l; Der eigene Frieden sollte bewahrt und notfalls wiederhergestellt werden - nicht der fremde Frieden in mehr oder weniger fernen Zonen; Beiträge zu weltweiter Stabilität und weltweirem Frieden wurden /.../ beschlossen I.../; Als Friedensdienstleister im früheren Jugoslawien schlüpfte das Bündnis zum ersten $\mathrm{Mal}$ in die neue Rolle /.../: Ist Bosnien die erste in einer langen Reihe künftiger NatoFricdensexpedisionen rumd um den Globus?; Wie vicle "Kriege für den Frieden« soll die Nato führen?

Okkasionalismen in textveflechtender Funktion, Text (2) 


\section{Bosnien ist kein Modell

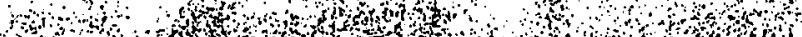

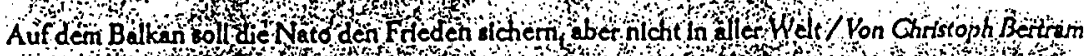

$3 \pi, 4$ on

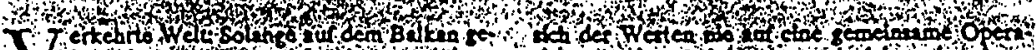

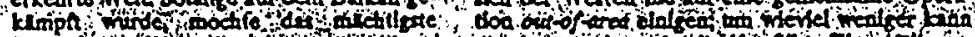

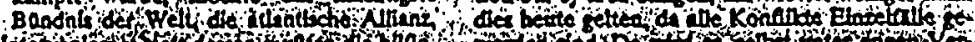

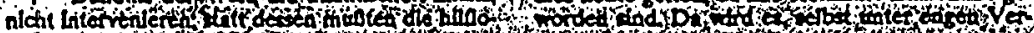

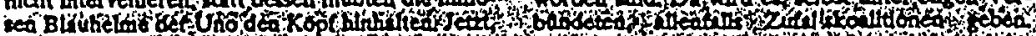

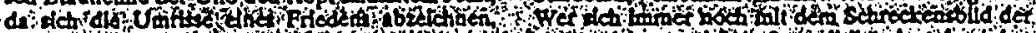

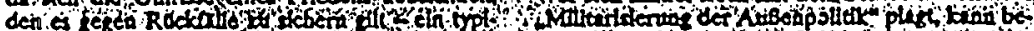

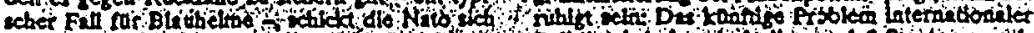

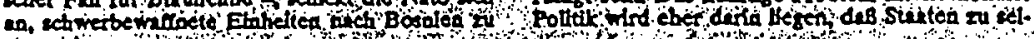
eatserided 3 of

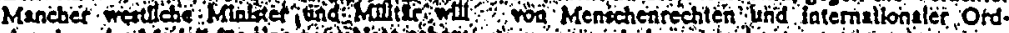

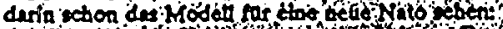

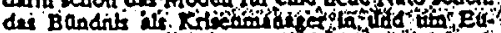

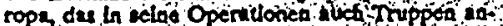

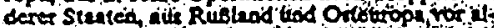

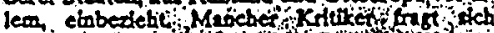

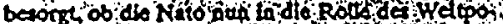

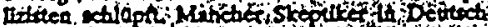

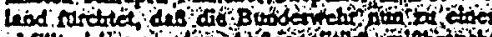

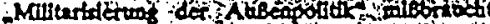

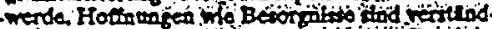

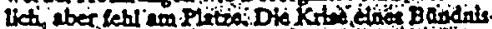

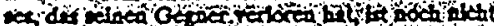

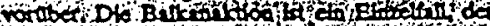

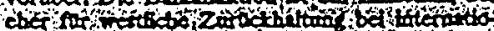

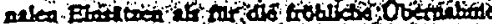

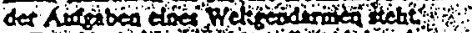

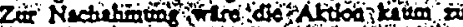

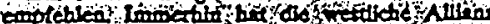

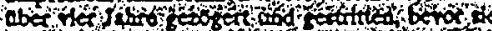

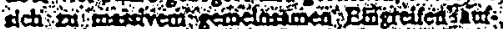

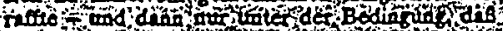

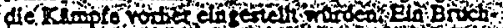

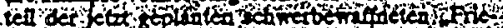

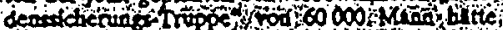

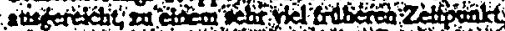

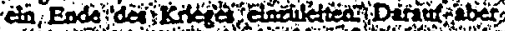

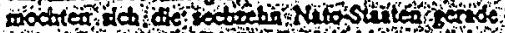

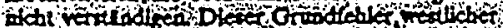

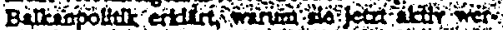

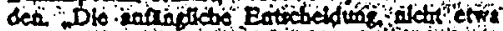

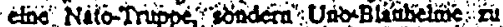

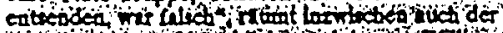

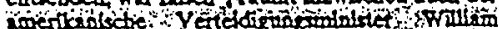

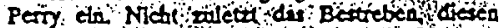

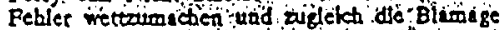
werticher Zeratritterbelt vergeséb zis tissen, gab - nun dea Anstoo, dio Blaubelme durct Oranhelitie abulose obgick die Nato-Soldaiea dóch el-

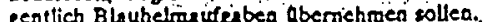

Die molisame Verrilindiguos zwivecher den Par. telen bezeugt dk ganze Fragwardigkeit des Vorbabeas die wertiche Allianz duret milliturebe Aufgeben.jeaseits der Bandatsereazea neu ra.

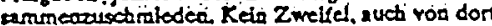
drobea Gefahreri. Aber Iri Oegensule zuider elo-

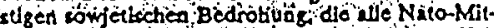

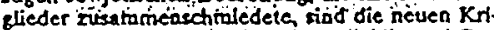
seo seeignet vie duseiniaderzudividlerea. Der Balkinkries bat des tur Oenage bestesen.

Im abrigen bul such hler in Blick in dic $\mathrm{Oe}^{-}$ schleblo: Scibrt la der Zell dea Kallea Krieger al Konnikte fensett der Baindoisgreaten vielfech weon such selicin zu Reche als Menlfertationen westostlchei Rtralitll podeutet werden, konnte

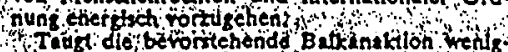

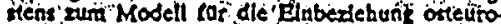

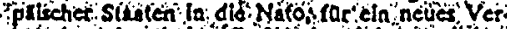

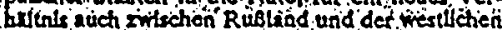

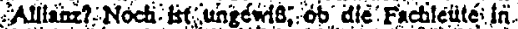

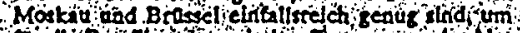

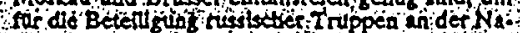

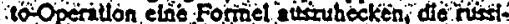

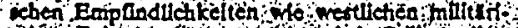

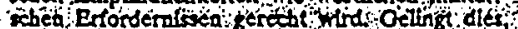

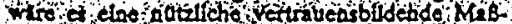

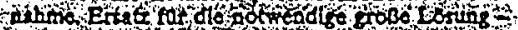

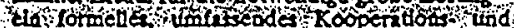

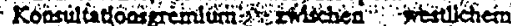

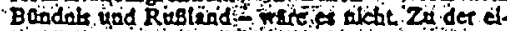

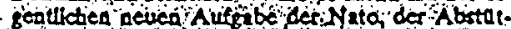

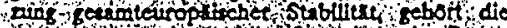

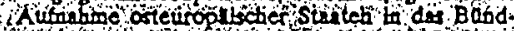

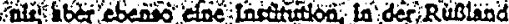

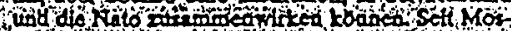

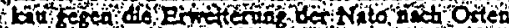

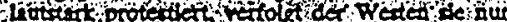

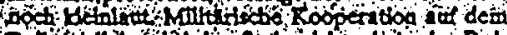

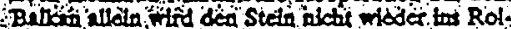

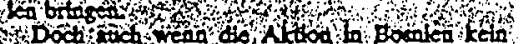

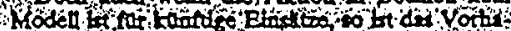

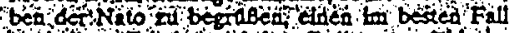

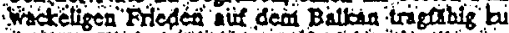

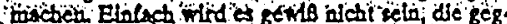

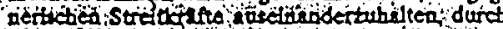

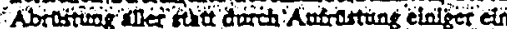

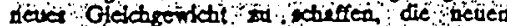

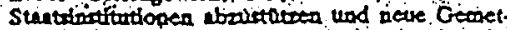
tel.20 rerhindern Dex. Gelledo verwint dic

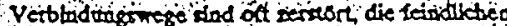

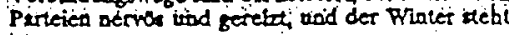
beror.

Noch. weth alemend roo welcber Dauer dic

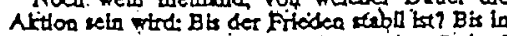
Amerika. Prisldenticturtrablen itattinden? Oder, we untinogst in Somette bis zu disem bluth gea. Zwischeafall det die Politiket hastig zum Ractrue blasen irst?

Besteht das Bondents die Herausforderung in ehemiligen Jugoslawien, vilre das tcboa eln gro Ger Erfols. Es whre eloc Kortektur fur vergangenes Verragen kelae. Oarnatie gegen-kanftiges Scheitera. Ohoe elne tunktlonlereade Nalo lst europatsche Subiliut njetet denkbar. Vielleiche funktionien das Bondnis auf dem Balkan endlich eln mal. Seine Zukunfi aber ist darnit-noch lange nieht gesicher.

Text (1), Die Zeit, Nr. 43 (20. Okt. 1995), S. 1 


\section{Bündnis}
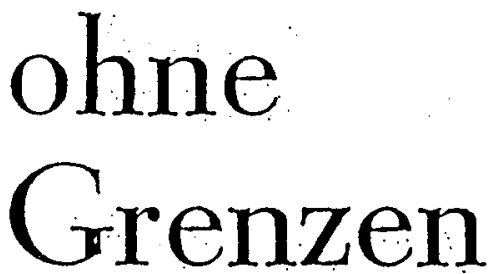

\author{
Die Nato in Bosnien: \\ Ein historischer Einschintt
}

\section{Von Theo Sommer}

I a dea alchsten Tagen wird die Vorbut roa einigen rigtausend Nato-Soldaten nach Bosnien auforecher. Das Expeditjonskorps der werlll. chen Allianz, verstarkt durch Soldaten aus zwolf anderen Landera einsctlieblich Rübliods soll deco beiklen Frieden verbtirgen den die sudslawischen Kriegsparteien vorige Wóhe geschlóssen bäben.

Im Ratselo daruber, was sich hinter dem Abkommen ron Dayton koakrel verbjigt urid was 1996 alles auf die Friedenstruppe zukommen mag, ist ein bistorisches Fartitit ziuni besehtet wordea: Die etlartische Alliant rostet dich auf das grobte milituriscbe Untemebmen ihrer 46jahrigen Geschichte - und mit der Bosnien-Mission geht sie weit aber ihren ursprongliebea Auftrag, ihren herkommlichen Altionsbereich hinaus.

Viet Jahrzehnte lang batte das Bundnis eine einzige Aulgabe: die Sowjetunion vor einem $A n$ griff auf die Mitgliedstaaten abzuschrecken und, falls es doch zu eirer Agsiession kommen sollte, "die Sicherheit des nordatlantischea Gebiets wiedetherzustellen". Artikel 5 des Nordataritikpakies begreazte das Vereidigungsareal auf das Tetritorium det Nato-Lunder. Kein Gedanke daran, daruber hinauszugreifen und int Einstien Jen-

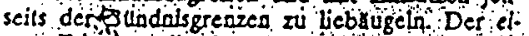
gerie Fntecln sollie bewabrt und notfalls wiederbergestell literden nicht der treonde Frieden in mehr odeituiniger lertien Zonen.
Nach de'h Fonde des Kaltea Krieges erweilerte die Allisnz' ihr Pflicbtenhefh Beitrlge zu weltwel: ter Subilitht und weliweltem Frieden wurdea auf

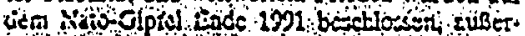

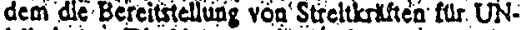
Missiodea. Die Nato wurde dámit eatgrenzt. Out of area or out of bustries hles unin die Parole: Erweitering Uen Einsitzöbletes oder SchlleBuirg

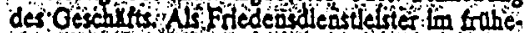

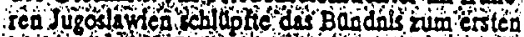
Mal to de acue Rolle, bislarg cingeglleder in dea Instanzenzug und die Befehlsketto der Vercinten

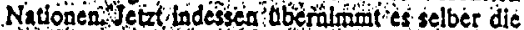
Regie. Ela Mandat des UN-Sichertieltsrates wird aur den papierenea Paravent abgeben, hinter dem die Nato in-elgener Zustlondigkeit, uf eigene Rechrung und Gelabr handelt.

Ist Bosnien die ente in einer langẹ Relhe ktinftiger Nato-Friedensexpeditionen rund um den Globus? Seitdem das Bundesverlassungsgericht deutscher Beteillgung an dejtel Udterach-

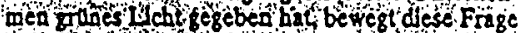

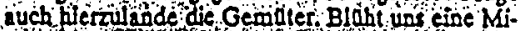
lianofering nun die Mensebetrech tspolith bistrer welthin cine soche der Oberieugung und des Mundwerts, endlich elpe gepanzerte Faust? Wie viele nKriege for déa Friedea" soll die Nato theren? An wie vielen mus de Bundesteptiblik sich beteiliger?

Die Befarchtungen sind so aberticben wie die Hoffnungen-In der Wirklichkeit unserer konflihtgeschuttelten Welt sind dem Inierventionismus Schrainked gesetze. Die dritte Welle der Oewalt. samkeit in 20. Jahrtundert - nach den Kriegen erst zwischen Staiten, dano zwischea Ideolopien nun die blutigen Nüselinandersetrüngen innerhalb cinzeloer Staaten - uberfordert alle. Es gibt zu viele Krisen. Das Eingreifen ist kostspielig. Und die Wirkung der Interyentionen bleibt fragwtordig oder nuchtis - siehe Somalis.

Am Eude bleibl nur ein brauchbarer Mabstab: das Inieresse - das ationale, das europlische, das atlantische Interesse, Es mag geographisch durch Nahe begrindet sein, politisch durch Sympathic, wirtschafilich dureh Abblogigkeit. In jedem Fall bedarf es der Ubereinkunft. Konsens jedoch fallt in westlichen BUndris sebwer, wo nicht $\mathrm{HaB}$ oder Furcht den Kitt der Gemeinsamkeit lielern, son. dem widersprilchliche moralische Regungen oder subjektive Nutzlichkẹitserwagungen waltea.

Der Begriff "Statsryson" Ist aus der Mode geraten. Es ist an der Zeit, ith wieder berrorzubolen: und rait : deutschem, curopăiscliem, allantischem Inhal ozúfule Die Purelbaune der

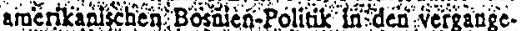

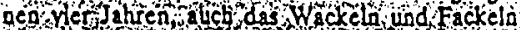

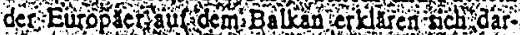
aus idahaleman ine holare Kriterie hat poch

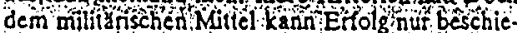
den sein, wenn der politische Zweck erkéntiar und einleuchitend dargestelli, es legitimiert:

Text (2), Die Zeit, Nr. 49 (1. Dez. 1995), S. 1 


\section{Winterfineden}

Nach vier Kriegswintera d Emmert dem oü dieBefriedung von außen Der kroatiehemaligen Jugoslawien ein Frieden lm sche Pils dent Franjo Tudjman dringt fünten Schnee und Nebel auf Gebros o hut schnelle Lut waftaung der Serben in

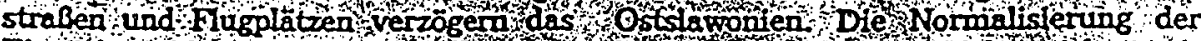

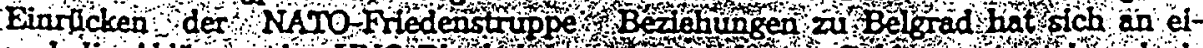

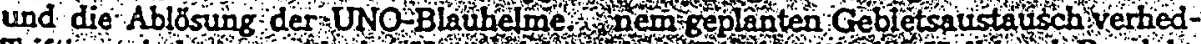

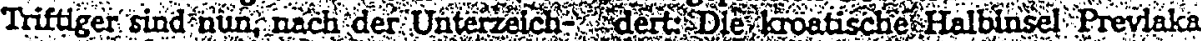

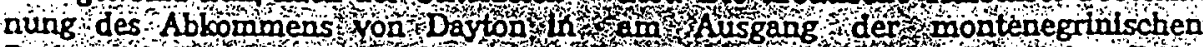

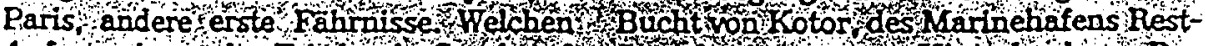

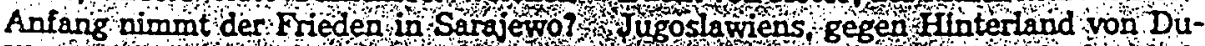

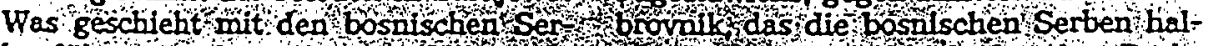
benführern und angelogten Kregster ton to teses thuschobjeld Ist aber Rech-

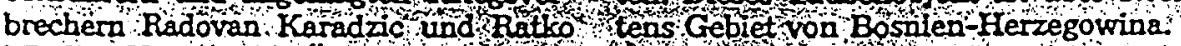
Madic? Konnat die Ubergangsregelung In Bonn soll an Wochenanfang eine für Ostslawopen om Ostrand Kroatens Konferenz den auftakt zu Rustungskonin Gang7 Verstandigen sich Belsad ond tolle fr tund tum Bosnien geben Das Zagreb euf gegenseitige A nerkennung ${ }^{2}$ Uhrwerk der Friten und Ybergange zum

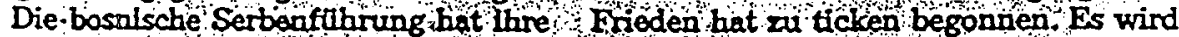

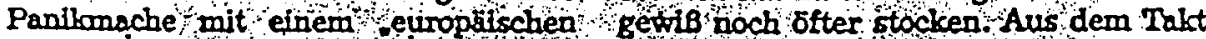
Beirut Sarajewo etwas gedïmpt Bine istes bisher noch picht geraten Der erste Tagung thres pariaments begannom foledenswinter aberwind in velen noch Sonntag bei noch ungeklarter Einstellung vier Kriegswinterngleicheo. Ki.

Text (3), Süddeutsche Zeitung, Nr. 291 (18. Dez. 1995), S. 4 
PRILOŽNOSTNO BESEDJE V MEDIJSKIH BESEDILIH.

\section{PRAGMALINGVISTIČNI VIDIKI RABE V BESEDILNI VRSTI ČASOPISNI KOMENTAR}

Prispevek obravnava teoretsko-metodološke predpostavke pragmatično naravnane besedilne in slogovne analize. $\mathrm{Ob}$ tem najprej problematizira besedoslovni pojem priložnostne besede in njenih definicij, nato predstavi pragmatično-sporočanjski model za opis in razlago besedila in sloga (Heusinger 1994) ter ga, modificiranega, uporabi za razlago in interpretacijo priložnostnih besed v izbranih časopisnih komentarjih iz nemškega nadregionalnega tiska. Pomembna ugotovitev je, da je za celostno opazovanje besedne ravnine besedila ter še posebej za analizo rabe priložnostnega besedja, ki izkazuje bogate slogovno-funkcionalne zmožnosti, potrebno upoštevati naslednje vidike: okoliščine sporočanja, sporočevalne vloge, govorna dejanja ter besedilno zgradbo vsakokratne besedilne vrste. Modificiran Heusingerjev model se pri tem izkazuje za uporabnega. 